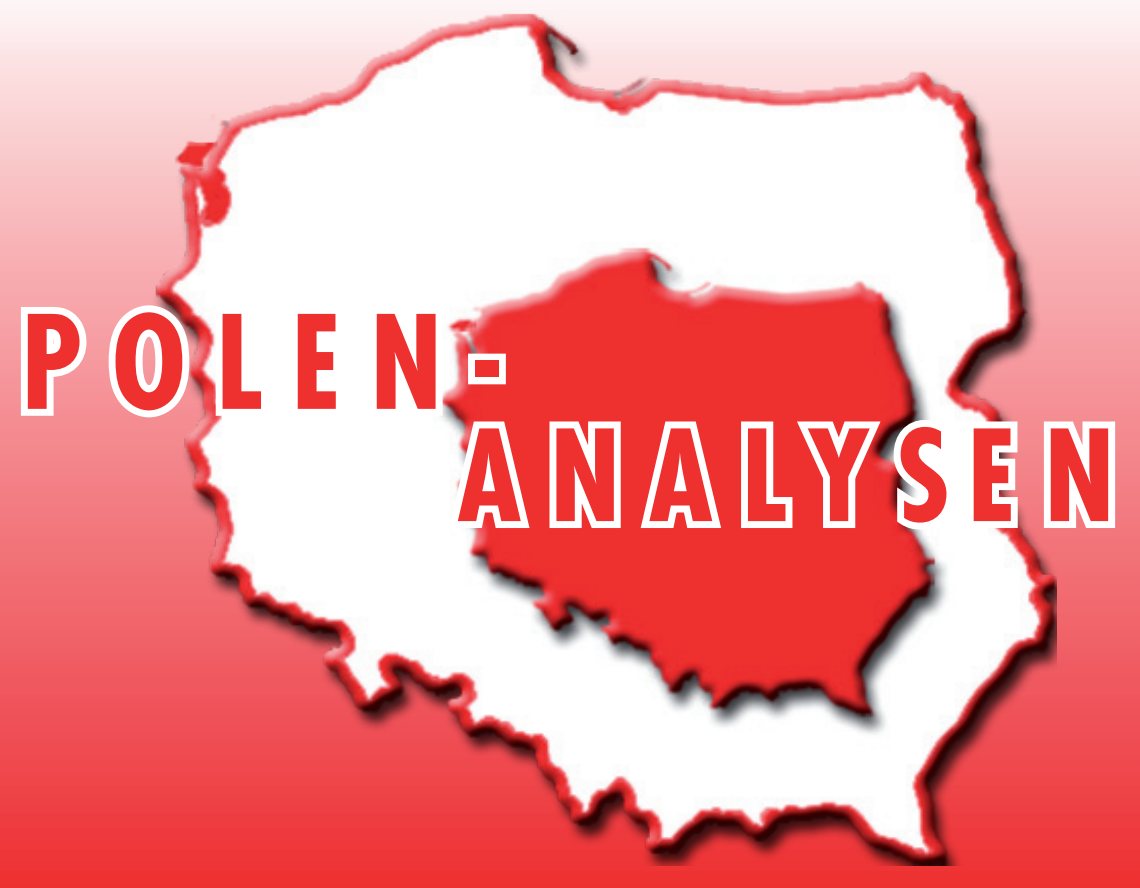

www.laender-analysen.de/polen

\title{
DIE WAHL ZUM EUROPÄISCHEN PARLAMENT IN POLEN
}

\section{ANALYSE}

Die Wahl zum Europäischen Parlament in Polen

Janusz A. Majcherek, Krakau

- TABELLEN UND GRAFIKEN ZUM TEXT

Umfragen zur und Ergebnis der Europawahl

\section{CHRONIK}

17. - 30. Juni 2014

Die nächste Nummer der Polen-Analysen erscheint nach der Sommerpause am Dienstag, den 2. September 2014.

Die Redaktion wünscht ihren Leserinnen und Lesern eine erholsame Sommerzeit.

Herausgegeben mit finanzieller Unterstützung der Stiftung für deutsch-polnische Zusammenarbeit

\section{DEUTSCHES} POLEN INSTITUT 


\title{
Die Wahl zum Europäischen Parlament in Polen
}

\author{
Janusz A. Majcherek, Krakau
}

\section{Zusammenfassung}

Bei der Wahl zum Europäischen Parlament konnte die regierende konservativ-liberale Bürgerplattform (Platforma Obywatelska - PO) einen minimalen Vorsprung vor der oppositionellen konservativ-nationalen Partei Recht und Gerechtigkeit (Prawo i Sprawiedliwość- PiS) erhalten. Die sinkende Unterstützung für die PO, die auch auf die Ermüdung der Wähler angesichts der langährigen politischen Dominanz dieser Partei zurückzuführen ist, wurde durch die Zustimmung zu den internationalen Aktivitäten der Regierung in der UkraineKrise aufgefangen. PiS kann insofern von einem Erfolg sprechen, da ihr Abstand zur PO nur minmal ist und sie ihren Besitzstand im Europäischen Parlament deutlich verbessert hat. Die Demokratische Linksallianz (Sojusz Lewicy Demokratycznej - SLD) sah sich damit konfrontiert, dass sich die sozial benachteiligten Wähler eher an PiS orientieren. Einen unerwarteten Wahlsieg erzielte die eskapistische Neue Rechte von Janusz Korwin-Mikke (Nowa Prawica Janusza Korwin-Mikke), die einen extremen ökonomischen Liberalismus mit einem kulturellen Ultrakonservatismus und Antietatismus verbindet, der sich antieuropäisch darstellt.

$\mathrm{N}_{\mathrm{n}}^{\mathrm{a}}$ ach einem schläfrigen Europa-Wahlkampf und bei niedriger Wahlbeteiligung bezwang die in Polen in der zweiten Legislaturperiode regierende konservativliberale Bürgerplattform (Platforma Obywatelska - PO) mit minimalem Abstand die oppositionelle konservativnationale Partei Recht und Gerechtigkeit (Prawo i Sprawiedliwość-PiS). Die Linke erlebte eine weitere Niederlage, die Sensation der Wahl war jedoch der exzentrische Ultralibertin Janusz Korwin-Mikke, dessen Gruppierung die 5-Prozent-Hürde mit über 7 Prozent deutlich überstieg und vier Mandate für sich gewann.

Wenige Monate vor der Wahl gab es die weit verbreitete Erwartung, dass sich der Wahlkampf auf landesspezifische und nicht auf europäische Probleme konzentrieren würde, nur wenige wählen gehen und die Oppositionspartei PiS siegen würde, was dem Überdruss in Anbetracht der langen Regierungszeit der $P O$ geschuldet gewesen wäre. Die Wahlbeteiligung ist in Polen bei allen Wahlen niedrig und bei den Europawahlen schon traditionell am niedrigsten (am höchsten ist sie bei den Präsidentschaftswahlen, die direkt und allgemein sind). In dieser Hinsicht haben sich die ursprünglichen Erwartungen erfüllt, nur knapp 24 Prozent der Wahlberechtigten gaben ihre Stimme ab. Die so hohe Wahlabsenz kontrastiert krass mit der sehr hohen Akzeptanz der Mitgliedschaft in der Europäischen Union in der polnischen Gesellschaft, mit der großen Zufriedenheit und dem großen Vertrauen gegenüber den Institutionen der EU, das deutlich höher als dasjenige ist, was den Politikern und staatlichen Organen im Land entgegen gebracht wird (eine Ausnahme ist das Amt des Staatspräsidenten). Eine der am stärksten pro-europäischen Gesellschaften in der EU ist also eine der am wenigsten bei der Wahl des Europäischen Parlaments engagierten (bei den vorangegan- genen EP-Wahlen war die Beteiligung nur ein Prozent höher). Diesen Widerspruch kann man mit der bei den Polen allgemein geringen Bereitschaft, die eigenen Ansichten und Überzeugungen in konkretem Handeln und persönlichem Engagement auszudrücken, erklären. Dies gilt auch für die geringe Beteiligung in Nicht-Regierungs-Organisationen, Gewerkschaften und bei Demonstrationen. Die Passivität in öffentlichen Angelegenheiten ist eine der charakteristischen Eigenschaften der heutigen Polen und die Wahl zum Europäischen Parlament hat diesen Befund bestätigt.

Auffällig ist auch, dass die Einführung des Euro in Polen im Wahlkampf nicht thematisiert wurde. Dies erklärt sich durch die ebenfalls überraschende Tatsache, dass die deutliche Mehrheit der pro-europäischen Polen die Einführung der europäischen Währung in Polen nicht will, was vor allem darauf beruht, dass die letzte Krise mit der Eurozone und eben ihrer Währung assoziiert wird: Die Wirtschaftskrise in Europa wird von vielen Polen als Krise der Eurozone wahrgenommen. Es herrscht die Überzeugung, dass es Polen gelang, sich vor dieser Krise dank einer eigenen Währung zu verteidigen, deren Kursschwankungen die ungünstigen Einflüsse der europäischen Wirtschaft amortisierten (der Fall des Polnischen Zloty erleichterte den Anstieg des Exports, auf den sich die Anti-Krisenpolitik stützte). Darüber hinaus stellen sich viele Polen recht naiv vor, dass die Übernahme der gemeinsamen Währung die Einführung vergleichbarer Preise wie in den anderen Euroländern bedeute, das heißt vor allem wie in Deutschland, wo die Preise aktuell mehrheitlich höher sind als in Polen. Die Politiker, die die Ablehnung der Polen gegenüber einer raschen Einführung des Euro kennen, haben dieses Thema im Wahlkampf vermieden. Ernstzunehmende Experten und Kommentatoren machen jedoch darauf 
aufmerksam, dass die wachsenden Bestrebungen Polens, eine größere Rolle in der Europäischen Union zu spielen, die sowohl aus dem Bevölkerungspotential resultieren (Polen stellt die sechstgrößte Bevölkerung in der EU) als sich auch aus dem steigenden wirtschaftlichen Potential ergeben, keine Substanz haben und nicht verwirklicht werden, solange Polen außerhalb der Eurozone bleibt, was im übrigen auch die Perspektive, wichtige Posten in der EU zu besetzen, einschränkt. Die Notwendigkeit, eine klare Strategie für den Beitritt zur Eurozone auszuarbeiten und vorzustellen, unterstrich vor den Europawahlen Staatspräsident Bronisław Komorowski - aber bitte erst nach den Wahlen!

Die Ereignisse in der Ukraine änderten jedoch die Thematik des Wahlkampfes und beeinflussten letztendlich das Wahlergebnis. Zum beherrschenden Thema wurde statt der erwarteten landesspezifischen Problematik die internationale und die Geopolitik. Die UkraineFrage hat für Polen und die Polen eine ganz wesentliche Bedeutung, denn die versuchte Unterordnung der Ukraine durch Russland würde bedeuten, dass Russland durch diese einen imperialen Status erlangt und den Weg des Expansionismus einschlägt, was für Polen eine tödliche Bedrohung darstellt, die schon manches Mal in der Geschichte Wirklichkeit geworden ist. Die Unabhängigkeit der Ukraine von Russland ist daher ein Bestandteil der polnischen Staatsräson. Der europäische Kontext, insbesondere die Beziehungen zwischen der EU und Russland und der EU und der Ukraine, hat für den aktuellen und den zukünftigen Status der Ukraine eine sehr große Bedeutung, weshalb er Gegenstand lebhafter Diskussionen und Debatten in der Zeit des Wahlkampfes wurde, der zeitgleich mit der Eskalation der Krise in der Ukraine stattfand. Dies war für die regierende $P O$ von Vorteil, denn Ministerpräsident Donald Tusk und Außenminister Radosław Sikorski waren in dieser Zeit intensiv auf internationaler Ebene tätig, trafen sich mit den wichtigsten europäischen Politikern, verkündeten weitere Initiativen, brillierten in den europäischen politischen Salons und waren deshalb die am häufigsten in den Medien präsenten polnischen Politiker, noch dazu in positiven Kontexten. Dies erhöhte ihre Umfragewerte, und die Unterstützung für die Ukraine und die Suche nach europäischen Verbündeten gegen Russland erlangten allgemeine Akzeptanz.

Die Opposition befand sich hier in der Defensive. Zum einen deshalb, weil die Versuche, der regierenden $P O$ verschiedene Fehler, Schwächen und Unterlassungen in der Innenpolitik vorzuhalten, keine Chance hatten, sich gegen die dominierende ukrainische Thematik durchzusetzen. Zum anderen, weil eben in dieser Thematik fast die gesamte Opposition eine ähnliche Position wie die Regierung einnimmt und sich weder durch
Andersartigkeit hervorheben konnte noch durch eigene Ideen und Initiativen. Es entstand der Eindruck, dass die ganze Initiative bei der Regierung liegt, die die Unterstützung der deutlichen Mehrheit der Bevölkerung und die Zustimmung der Opposition erfuhr.

Dies führte zur Umkehr eines Trends, der seit etlichen Monaten vor den Wahlen einen anhaltenden Vorsprung der oppositionellen PiS und eine hohe Wahrscheinlichkeit, dass diese die Europawahlen gewinnen würde, anzeigte, was wiederum die Unterbrechung der fatalen Pechsträhne von PiS gewesen wäre, die sich in den Niederlagen bei sieben aufeinander folgenden Wahlen (des Parlaments, des Staatspräsidenten, Kommunalwahlen und die letzen Europawahlen) niedergeschlagen hatte. Das Ergebnis der aktuellen Wahlen zum Europäischen Parlament galt also als Prognose vor den Kommunalwahlen im Herbst dieses Jahres und den Präsidentschafts- und den Parlamentswahlen im kommenden Jahr.

\section{Der minimale Sieg der PO}

Das Wahlergebnis der aktuellen Europawahl ist nicht eindeutig, obgleich es symbolische Bedeutung hat, da die $P O$ doch wieder gewonnen hat und die Serie ihrer Wahlsiege und der Niederlagen von $P i S$ verlängerte. Es war dies jedoch ein minimaler Sieg, denn es waren kaum 25.000 Stimmen von den 7 Mio. abgegebenen, das heißt weniger als 0,5 Prozent. Das bedeutet, dass die $P O$ und PiS beide 19 Sitze im Europäischen Parlament erhalten haben (insgesamt stehen Polen 51 Mandate zu). In ihrer Fraktion nimmt die $P O$ allerdings eine ungleich stärkere Position ein, denn auch wenn sie durch das aktuelle Wahlergebnis sechs Mandate verloren hat (vorher hatte sie 25 Sitze inne), gehören ihre Abgeordneten zur Siegerfraktion, der Fraktion der Europäischen Volkspartei (EVP), wo sie der stärkste Bündnispartner der CDU sind und mit wichtigen Posten und entsprechendem Einfluss rechnen können. Darüber hinaus haben sie die Unterstützung der vier Europaabgeordneten der Polnischen Bauernpartei (Polskie Stronnictwo Ludowe $P S L)$, mit der sie in Polen die Regierungskoalition bilden und die ebenfalls zur EVP gehört. Der ehemalige polnische Ministerpräsident Jerzy Buzek war vor Martin Schulz Präsident des Europäischen Parlaments und Janusz Lewandowski der sehr wichtige Haushaltskommissar. In der beginnenden Amtsperiode sind die Ambitionen der Polen nicht geringer.

Die $P O$ ist eine proeuropäische Partei, aber mit vielen Strömungen und folglich zurückhaltend und vorsichtig gegenüber Projekten einer stärkeren Integration innerhalb der EU, die nicht alle ihre Politiker unterstützen. Mit der CDU läuft die Verständigung und Zusammenarbeit gut. 


\section{Der Erfolg von PiS}

PiS kann auch von einem Erfolg sprechen, denn sie hat nur minimal gegenüber der $P O$ verloren und ihren Besitzstand im Europäischen Parlament deutlich verbessert. Bei der vorangegangenen Wahl hatte sie nur 15 Mandate erhalten, zudem verließen einige Europaabgeordnete während der Amtszeit die Partei und ihre Fraktion zerfiel. Dennoch ist die Position von PiS auch weiter schwach, denn sie gehört zur marginalen Fraktion der Europäischen Konservativen und Reformisten (EKR), ebenso wie die britischen Konservativen, die außerdem im Kampf mit der UKIP eine Niederlage erfuhren (zur EKR gehört außerdem die Alternative für Deutschland). Das Verhältnis von $P i S$ zu den Torys ist kompliziert und schwierig, denn diese betrieben letztens eine Antiimmigrationskampagne, die auch gegen die auf der Insel arbeitenden Polen gerichtet war, und das nahende Referendum über den Verbleib Großbritanniens in der Europäischen Union wird die Propaganda gegen die Immigranten sicherlich noch verschärfen. Hinzu kommt, dass die britischen Konservativen eine Beschränkung der Größe des europäischen Haushaltes fordern, dessen größter Nutznießer Polen ist. Im Europäischen Parlament haben die Abgeordneten von $P i S$ also keine starken und treuen Verbündeten, daher zählen sie im Grunde nicht und haben keine Chance, eine bedeutende Rolle zu spielen. Das Ergebnis der Europawahl behandelt die Parteiführung aber als gute Prognose für die diesjährigen Kommunalwahlen in Polen und die Parlamentswahlen im kommenden Jahr (bei den Präsidentschaftswahlen hat kein Politiker von PiS die Chance, ein erfolgreicher Rivale des populären und geschätzten Staatspräsidenten Bronisław Komorowski zu werden, der wohl ganz sicher noch einmal kandidieren wird). Die Unterstützung durch die Wählerschaft auf einem mit der $P O$ vergleichbaren Niveau nährt die Hoffnung von PiS, der Bürgerplattform die Regierungsverantwortung abnehmen zu können.

PiS ist eine europaskeptische Partei, aber keine antieuropäische, wenn auch eine antiföderalistische und gegen eine stärkere Integration. Sie unterstreicht die Notwendigkeit, die Souveränität der EU-Mitgliedstaaten zu bewahren und wiederholt den alten Begriff de Gaulles des »Europa der Vaterländer", was als Kooperation der Nationalstaaten zu verstehen ist.

\section{Die Niederlage der SLD}

Eine Niederlage erfuhr zum wiederholten Male die polnische Linke. Die vom ehemaligen Ministerpräsidenten Leszek Miller geführte Demokratische Linksallianz (Sojusz Lewicy Demokratycznej - SLD), die im Wahlkampf von Martin Schulz unterstützt wurde, erhielt knapp 10 Prozent der Stimmen und fünf Abgeordne- tenmandate. Die Krise der polnischen Linken dauert also weiter an und es helfen ihr auch keine Image- oder Propagandamaßnahmen, wozu auch Versuche gehörten, Stars aus dem Showbusiness auf die Wahllisten zu setzen. Die grundlegende Ursache dafür ist das Fehlen eines erkennbaren und sinnvollen programmatischen und ideellen Angebots. In wirtschaftlichen Schlüsselfragen hat die Linke nichts anzubieten, sie wiederholt Allgemeinplätze vom Schutz der Schwächsten, sozialer Gerechtigkeit und dem Kampf gegen die Arbeitslosigkeit oder soziale Ausgrenzung. Dabei kam die polnische Wirtschaft gut in der Krise zurecht und befindet sich aktuell auf einer Welle hohen Wachstums (über 3,5 Prozent im 1. Quartal 2014 mit Indizien, dass es so weitergehen wird), was nicht den Boden für soziale Parolen bereitet. Die Linke hat außerdem die Entstehung und die Stärkung der Mittelschicht in Polen verpasst, die nun beginnt, das öffentliche Leben zu dominieren und gleichgültig gegenüber den Slogans für Arbeitslose oder schlecht verdienende Arbeiter ist, die seit Jahren von der Linken wiederholt werden. Außerdem wurde die sozial orientierte und fordernde Wählerschaft in einem deutlichen Maße von PiS abgefangen, was mit dem gesamteuropäischen Trend übereinstimmt, dass sich die Unzufriedenen an der Rechten orientieren. In Polen sind darüber hinaus die Ärmeren mit einem niedrigeren sozialen und materiellen Status im Allgemeinen eng mit der katholischen Kirche verbunden, die in sittlichen Fragen konservativ und weit von den weltanschaulichen Forderungen der Linken entfernt ist. Die Slogans der Linken landeten also in einem gesellschaftlichen Vakuum.

Mit einem Fiasko endete das speziell für die Europawahl initiierte Projekt einer alternativen Mitte-LinksFormation, die sich auf Janusz Palikot und den ehemaligen Staatspräsidenten Aleksander Kwaśniewski stützte. Palikot, ehemaliger PO-Abgeordneter, war der Urheber der großen Überraschung vor drei Jahren, als er an der Spitze der ad hoc gegründeten Palikot-Bewegung (Ruch Palikota) in den Parlamentswahlen über 10 Prozent erlangte und damit die drittgrößte Fraktion stellte. Deren Hauptmerkmal war, außer der Mitgliedschaft der ersten Transsexuellen im Sejm, ein scharfer Antiklerikalismus, der allerdings immer sonderbarere und geschmacklosere Form annahm und mit einer völlig unklaren Haltung zu anderen Fragen, insbesondere wirtschaftlichen, verknüpft war. Die Schirmherrschaft des ehemaligen und weiterhin allgemein geschätzten Staatspräsidenten Aleksander Kwaśniewski und die von ihm auf die Wahlliste beförderten politischen Freunde (darunter auch bisherige Europaabgeordnete) stärkten diese Formation nicht, sondern verwischten ihr Profil, was ihr Angebot noch unklarer werden ließ. Die Wähler gaben ihrer Desorientierung und Enttäuschung Aus- 
druck, indem sie die Gruppierung unter dem Namen Europa Plus - Deine Bewegung (Europa Plus Twój Ruch) unter der 5-Prozent-Hürde landen ließen, so dass sie keine Mandate für das Europäische Parlament erlangte. Das Wahlbündnis hat sich bereits aufgelöst.

\section{Ein Exzentriker sorgt für eine Sensation}

Die Überraschung und Sensation der Wahl zum Europäischen Parlament wurde der exzentrische Veteran Janusz Korwin-Mikke. Dieser 72-jährige Politiker und vielschreibende Publizist (und gefragte Bridge-Spieler), politisch aktiv und für seine antikommunistische Tätigkeit schon 1964 mit Repressionen konfrontiert, inhaftiert auch 1968, begründete in den 1970er Jahren die organisierte antikommunistische Opposition mit, und zwar an ihrem extrem wirtschaftsliberalen und konservativem Rand, fasziniert von Margret Thatcher und Ronald Reagan. An der Spitze unterschiedlicher Gruppierungen nahm er am politischen Leben auch nach dem Zusammenbruch des kommunistischen Systems teil, geriet aber als unberechenbarer und nicht ernstzunehmender Sonderling ins Abseits. Ein solches Image verdankte er nicht nur seinem fortgeschrittenen Libertarianismus und Engagement für immer kuriosere Kampagnen (zum Beispiel gegen die Gurtpflicht beim Autofahren als Beschränkung der persönlichen Freiheit) sowie seinen propagierten empörenden Ansichten (beispielsweise über die Entbehrlichkeit des Frauenwahlrechtes, weil Frauen ähnlich wie die Männer wählen und eine Doppelung der Stimmen nicht notwendig sei), sondern auch seinem Aussehen und seiner Lebensart (immer mit Fliege, spitzer Zunge und charakteristischem Stottern). Über Jahre hatte er eine Gruppe faszinierter Anhänger, die treffender als Jünger zu bezeichnen sind, aber bei keiner Wahl überschritt er die 5-Prozent-Hürde. Gegenwärtig steht er an der Spitze der Partei Kongress der Neuen Rechten (Kongres Nowej Prawicy), deren politisch-ideelles Profil und Programm der amerikanischen Tea-Party-Bewegung am nächsten kommt. Sie verbindet einen extremen ökonomischen Liberalismus mit einem kulturell-sittlichen Ultrakonservatismus und Antietatismus, der sich auch als antieuropäisch offenbart. In den vergangenen Jahren erhielt er insbesondere die Sympathien der jüngsten Wähler (und unter den jungen Polen, die das Wahlalter noch nicht erlangt haben, ist er der beliebteste Politiker) und dank ihrer Stimmen trug er aktuell einen Wahlsieg davon. Dies ist insofern überraschend, als Korwin-Mikke weder ein junger noch ein neuer Akteur in der polnischen Politik ist. Die Unterstützung der Jugend verdankt er nicht nur deren natürlicher Bereitschaft, einfache und radikale Lösungen zu akzeptieren, sondern auch seiner AntiestablishmentRhetorik, seiner beständigen Existenz außerhalb der
Hauptströmung der polnischen Politik und auch dem Umstand, dass er nie irgendwelche Posten besetzt hat, was ihm erlaubt, sich als unabhängiger, in nichts verwickelter, nicht kompromittierter und alternativer Politiker darzustellen. Auch die Verwunderung hervorrufenden Verständniserklärungen gegenüber Putins Aktivitäten bestätigten seine Andersartigkeit, seinen Charakter und seine Unabhängigkeit vor dem Hintergrund der anderen polnischen Politiker und Gruppierungen. In der Unterstützung für ihn kam außerdem die den jungen Wählern eigene Neigung zu Trotz und Albernheit zum Ausdruck, ähnlich der, die in Italien dem Komiker Beppe Grillo Erfolg beschert hatte. Diese Analogie ist auch insofern begründet, als Korwin-Mikke manchmal den Eindruck eines Possenreißers macht, der in seinen Äußerungen und Handlungen nicht ganz ernst zu nehmen ist. Vor dem Hintergrund der radikalen, antieuropäischen und Antiestablishment-Rechten in Europa sind die von Korwin-Mikke erlangten 7 Prozent allerdings ein relativer und moderater Erfolg und eher als ein unerwartetes Übersteigen der 5-Prozent-Hürde nach vielen Jahren zu werten, als dass davon auszugehen ist, dass er tatsächlich Einfluss und Positionen in der polnischen und umso weniger in der europäischen Politik erlangen wird.

Die Vertreter der nationalistischen Rechten, die seit einigen Jahren immer lautstarker ihre Anwesenheit in der öffentlichen Sphäre manifestieren, erhielten die Unterstützung der Polen nicht. Die Nationale Bewegung (Ruch Narodowy) hat mit 1,4 Prozent der Stimmen eine Wahlniederlage eingefahren und den letzten Platz auf der Liste der gesamtpolnischen Gruppierungen, die an den Wahlen zum Europäischen Parlament teilnahmen, belegt. Der radikale Nationalismus findet bei den Polen keine politische Unterstützung und spielt keine politische Rolle.
Alles im Lot?
Die Wahl zum Europäischen Parlament führte in Polen zu keiner Erschütterung und führte nicht einmal zu einer Veränderung der politischen Situation, die sich mit den Staaten vergleichen ließe, in denen unerwartete Ent- scheidungen fielen und extreme und radikale Parteien triumphierten. Zum wiederholten Male gewann die $P O$, deren sinkende Unterstützung zum Teil das natürli- che Ergebnis der Ermüdung und Langeweile angesichts ihrer langjährigen politischen Dominanz ist. Auf den Fersen folgt ihr wie schon seit Jahren PiS. Die Rivalität dieser beiden Gruppierungen ist eine beständige Achse der polnischen Politik, die die übrigen Parteien in die zweite Reihe schiebt. Zum Teil ergibt sich eben daraus die durch die Europawahl bestätigte Schwäche der polni- schen Linken, die seit Jahren in der Krise und in Kraftlo- sigkeit versunken ist, nicht in der Lage, irgendeine inte- 
ressante und attraktive Botschaft zu formulieren und den polnischen Wählern vorzustellen. Als beständig erwies sich außerdem, entgegen vieler Vorhersagen, die Präsenz der PSL in der polnischen Politik, die ihre treue ländliche Wählerschaft hat. Diese garantiert ihr 7 bis 8 Prozent Unterstützung und damit eine große Befähigung, eine Regierungskoalition einzugehen. Begünstigt wird die stabile Position der PSL durch die Zufriedenheit der polnischen Landwirte über die Mitgliedschaft in der Europäischen Union, die trotz ihrer Ängste und Widerstände vor dem EU-Beitritt seit längerem feststellbar ist. Die dauerhaften Zuzahlungen und Subventionen sowie die aus EU-Mitteln finanzierten Infrastrukturinvestitionen machen die Landwirte und die ländliche Bevölkerung zu den größten Nutznießern der Mitgliedschaft in der EU. Dank ihrer charakteristischen Elastizität und ihrer Cleverness (von Boshaften wird dies mit der bäuerlichen Herkunft assoziiert) war die PSL schon eine Koalition mit der Linken eingegangen, jetzt ist es die $P O$ und nicht ausgeschlossen ist ihre Beteiligung auch an anderen Konfigurationen.

Die Wahl zum Europäischen Parlament hat also keine grundsätzlichen Veränderungen der polnischen politischen Situation nach sich gezogen. Viel größere Folgen kann die Affäre um die Veröffentlichung vertraulicher Gespräche führender polnischer Politiker einige Wochen nach der Wahl nach sich ziehen - das aber ist eine andere Angelegenheit.

Übersetzung aus dem Polnischen: Silke Plate

Über den Autor:

Prof. Dr. habil. Janusz A. Majcherek ist Leiter des Lehrstuhls für Soziologie an der Pädagogischen Universität (Uniwersytet Pedagogiczny) in Krakau und Kommentator politischer Themen in den polnischen Medien.

\section{Umfragen zur und Ergebnis der Europawahl}

\section{Tabelle 1: Die Teilnahme an der Wahl zum Europäischen Parlament}

\begin{tabular}{|c|c|c|c|}
\hline \multirow{2}{*}{$\begin{array}{l}\text { Unterstützen Sie die Mit- } \\
\text { gliedschaft Polens in der } \\
\text { Europäischen Union oder sind } \\
\text { Sie dagegen? }\end{array}$} & \multicolumn{3}{|c|}{ Beabsichtigen Sie, an der Wahl zum Europäischen Parlament teilzunehmen? } \\
\hline & $\begin{array}{l}\text { Ich nehme ganz sicher } \\
\text { teil. }\end{array}$ & Ich weiß noch nicht. & $\begin{array}{l}\text { Ich nehme eher nicht } \\
\text { teil. }\end{array}$ \\
\hline Anhänger & $43 \%$ & $28 \%$ & $29 \%$ \\
\hline Gegner & $29 \%$ & $22 \%$ & $50 \%$ \\
\hline schwer zu sagen & $29 \%$ & $21 \%$ & $50 \%$ \\
\hline
\end{tabular}


Tabelle 2: Die Teilnahme an der Wahl zum Europäischen Parlament im Mai 2014

\begin{tabular}{|l|c|c|c|}
\hline Wählerschaften nach Parteien* & \multicolumn{2}{|c|}{$\begin{array}{c}\text { Beabsichtigen Sie, an der Wahl zum Europäischen Parlament teilzunehmen? } \\
\text { (\%) }\end{array}$} \\
\hline PiS & $\begin{array}{c}\text { Ich nehme ganz sicher } \\
\text { teil. }\end{array}$ & $\begin{array}{c}\text { Ich weiß noch nicht. } \\
\text { Ich nehme eher nicht } \\
\text { teil. }\end{array}$ \\
\hline PO & 76 & 18 & 6 \\
\hline Nowa Prawica Janusza Korwin- & 75 & 21 & 4 \\
Mikke* & 71 & 21 & 8 \\
\hline SLD & 67 & 16 & 13 \\
\hline PSL** & 62 & 25 & 12 \\
\hline unentschlossene & 69 & 19 & 58 \\
\hline $\begin{array}{l}\text { Nichtwähler bei eventuellen Par- } \\
\text { lamentswahlen }\end{array}$ & 8 & 35 & 13 \\
\hline
\end{tabular}

PiS - Prawo i Sprawiedliwośc/Recht und Gerechtigkeit; PO - Platforma Obywatelska/Bürgerplattform; Nowa Prawica Janusza Korwin-Mikke/Neue Rechte von Janusz Korwin-Mikke; SLD - Sojusz Lewicy Demokratycznej/Demokratische Linksallianz; PSL - Polskie Stronnictwo Ludowe/Polnische Bauernpartei

${ }^{*}$ Nach Parteipräferenzen bei eventuellen Wablen zum Sejm.

**Aufgrund der geringen Anzahl derer, die diese Partei unterstützen würden, muss das Ergebnis mit Vorsicht interpretiert werden. Quelle: CBOS Nr. 71/2014 Wybory do parlamentu europejskiego [Die Wabl zum Europäischen Parlament]. Warszawa 05/2014. $<$ www.cbos.pl>

Tabelle 3:Ergebnisse der angetretenen Parteien und Wahlbündnisse bei der Europawahl am 25. Mai 2014

\begin{tabular}{|l|c|}
\hline \multicolumn{1}{|c|}{ Parteien/Wahlbündnisse } & Stimmenanteil \\
PO & $32,13 \%$ \\
PiS & $31,78 \%$ \\
\hline SLD & $9,44 \%$ \\
Nowa Prawica Janusza Korwin-Mikke & $7,15 \%$ \\
\hline PSL & $6,80 \%$ \\
\hline Solidarna Polska Zbigniewa Ziobra & $3,98 \%$ \\
Wahlbündnis Europa Plus Twój Ruch & $3,58 \%$ \\
\hline Polska Razem Jarosława Gowina & $3,16 \%$ \\
\hline Ruch Narodowy & $1,40 \%$ \\
Partia Zieloni & $0,32 \%$ \\
Demokracja Bezpośrednia & $0,23 \%$ \\
Samoobrona & $0,04 \%$ \\
Wahlbeteiligung insgesamt & $23,82 \%$ \\
\hline
\end{tabular}

PO - Platforma Obywatelska/Bürgerplattform; PiS - Prawo i Sprawiedliwość/Recht und Gerechtigkeit; SLD - Sojusz Lewicy Demokratycznej/Demokratische Linksallianz; Nowa Prawica Janusza Korwin-Mikke/Neue Rechte von Janusz Korwin-Mikke; PSL - Polskie Stronnictwo Ludowe/Polnische Bauernpartei; Solidarna Polska Zbigniewa Ziobra/Solidarisches Polen von Zbigniew Ziobro; Europa Plus Twój Ruch/Europa Plus - Deine Bewegung; Polska Razem Jarostawa Gowina/Polen Gemeinsam von Jarosław Gowin; Ruch Narodowy/ Nationale Bewegung; Partia Zieloni/Partei Die Grünen; Demokracja Bezpośrednia/Direkte Demokratie; Samoobrona/Selbstverteidigung Quelle: Obwieszczenie Państwowej Komisji Wyborczej z dnia 26 maja 2014r. o wynikach wyborów postów do Parlamentu Europejksiego przeprowadzonych $w$ dniu 25 maja 2014r. [Bekanntmachung der Staatlichen Wablkommission vom 26. Mai 2014 über die Ergebnisse der Abgeordnetenwahlen zum Europäischen Parlament am 25. Mai 2014], <http://pkw.gov.pl/g2/oryginal/2014_05/966 be725a42d277a1cd313ba2af21e6f.pdf> (abgerufen am 30.06.2014) 
Tabelle 4: Fraktionszugehörigkeiten der polnischen Abgeordneten des Europaparlaments

\begin{tabular}{|c|c|c|c|c|}
\hline $\begin{array}{l}\text { Fraktion der Euro- } \\
\text { päischen Volkspartei } \\
\text { (EVP) }\end{array}$ & $\begin{array}{l}\text { Fraktion der Pro- } \\
\text { gressiven Allianz der } \\
\text { Sozialdemokraten } \\
\text { (S\&D) }\end{array}$ & $\begin{array}{l}\text { Europäische Konser- } \\
\text { vative und Refor- } \\
\text { misten (EKR) }\end{array}$ & sonstige & insgesamt \\
\hline $\begin{array}{c}23 \text { (davon: PO: } 19 \text {, } \\
\text { PSL: 4) }\end{array}$ & $5(\mathrm{SLD})$ & 19 (PiS) & $\begin{array}{c}4 \text { (Nowa Prawica } \\
\text { Janusza Korwin- } \\
\text { Mikke) }\end{array}$ & 51 \\
\hline
\end{tabular}

PO-Platforma Obywatelska/Bürgerplattform; PSL - Polskie Stronnictwo Ludowe/Polnische Bauernpartei; SLD - Sojusz Lewicy Demokratycznej/Demokratische Linksallianz; PiS - Prawo i Sprawiedliwość/Recht und Gerechtigkeit; Nowa Prawica Janusza Korwin-Mikkel Neue Rechte von Janusz Korwin-Mikke

Quelle: Europäisches Parlament: Ergebnisse der Europawahl 2014. Sitze nach Mitgliedstaat. Verteilung der nationalen politischen Parteien und Gruppen.<http://www.ergebnisse-wahlen2014.eu/de/seats-member-state-absolut.html> (abgerufen am 1.07.2014)

Grafik 1: Wen würden Sie wählen, wenn am nächsten Sonntag Parlamentswahl wäre? (\%)

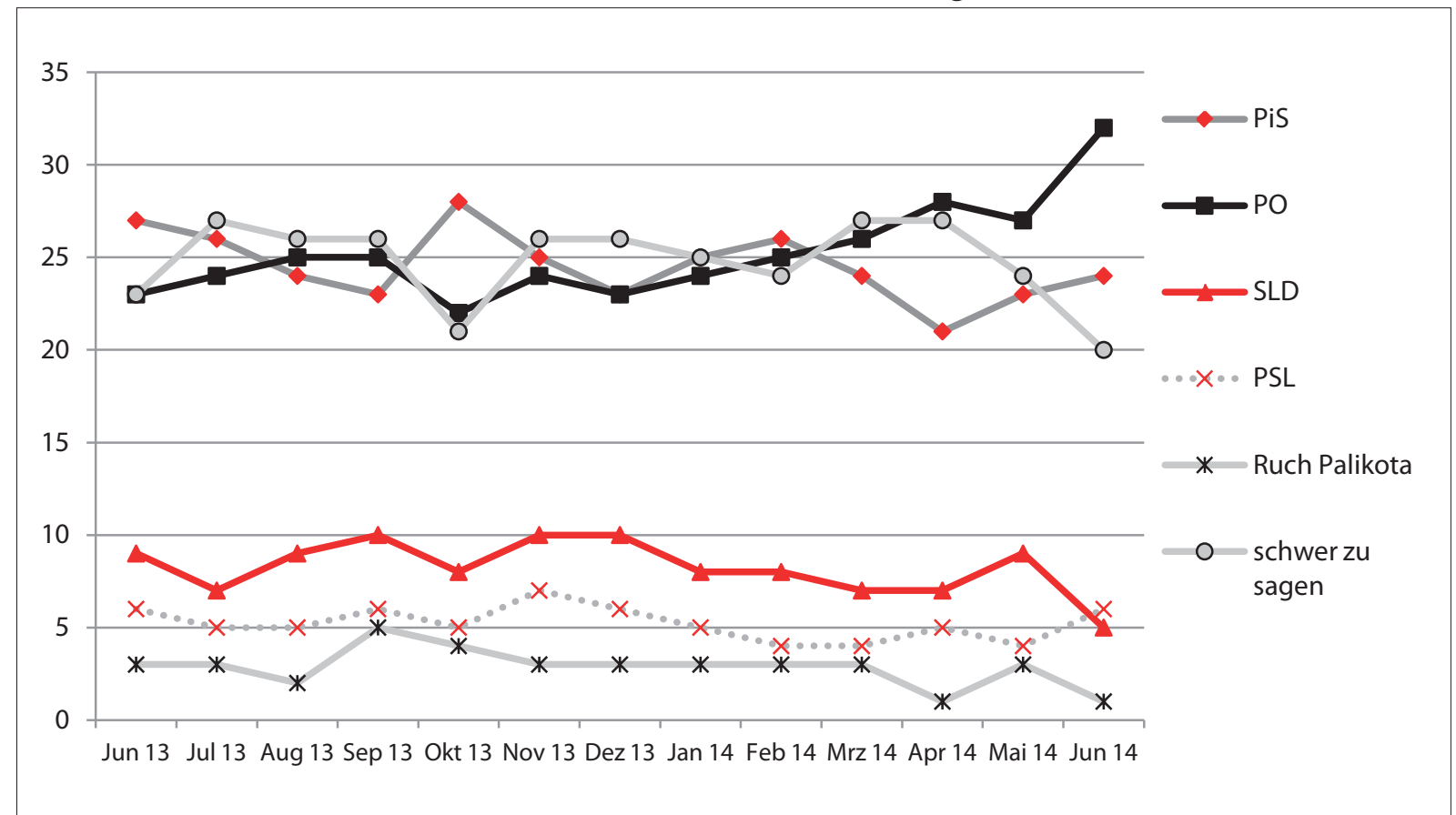

Anm.: Berücksichtigt wurden die Antworten derer, die angaben, zur Wahl gehen zu wollen.

PiS - Prawo i Sprawiedliwość/Recht und Gerechtigkeit; PO - Platforma Obywatelska/Bürgerplattform; SLD -Sojusz Lewicy Demokratycznej/Demokratische Linksallianz; PSL - Polskie Stronnictwo Ludowe/Polnische Bauernpartei; Ruch Palikota/Palikot-Bewegung Quelle: CBOS Nr. 85/2014 Preferencje partyjne po wyborach do parlamentu europejskiego [Parteipräferenzen nach der Wahl zum Europäischen Parlament]. Warszawa 06/2014. <www.cbos.pl> 
Tabelle 5: Wen würden Sie wählen, wenn am nächsten Sonntag Parlamentswahl wäre? (\%)

\begin{tabular}{|c|c|c|c|c|c|c|c|c|c|c|c|c|c|}
\hline & $06 / 13$ & $07 / 13$ & $08 / 13$ & $09 / 13$ & $10 / 13$ & $11 / 13$ & $12 / 13$ & $01 / 14$ & $02 / 14$ & $03 / 14$ & $04 / 14$ & $05 / 14$ & $06 / 14$ \\
\hline $\mathrm{PO}$ & 23 & 24 & 25 & 25 & 22 & 24 & 23 & 24 & 25 & 26 & 28 & 27 & 32 \\
\hline PiS & 27 & 26 & 24 & 23 & 28 & 25 & 23 & 25 & 26 & 24 & 21 & 23 & 24 \\
\hline PSL & 6 & 5 & 5 & 6 & 5 & 7 & 6 & 5 & 4 & 4 & 5 & 4 & 6 \\
\hline SLD & 9 & 7 & 9 & 10 & 8 & 10 & 10 & 8 & 8 & 7 & 7 & 9 & 5 \\
\hline $\begin{array}{l}\text { Nowa Prawica } \\
\text { Janusza Korwin- } \\
\text { Mikke }\end{array}$ & 2 & 2 & 2 & 2 & 4 & 1 & 2 & 3 & 4 & 3 & 5 & 5 & 5 \\
\hline Solidarna Polska & 2 & 1 & 2 & 0 & 2 & 1 & 2 & 2 & 2 & 2 & 1 & 1 & 3 \\
\hline Ruch Palikota & 3 & 3 & 2 & 5 & 4 & 3 & 3 & 3 & 3 & 3 & 1 & 3 & 1 \\
\hline $\begin{array}{l}\text { PJN/Polska } \\
\text { Razem Jarosława } \\
\text { Gowina }\end{array}$ & $1^{*}$ & $1^{*}$ & $1^{*}$ & $0^{*}$ & $0^{*}$ & $1^{*}$ & $1^{*}$ & 3 & 2 & 2 & 3 & 2 & 1 \\
\hline $\mathrm{PPP}$ & 1 & 0 & 0 & 0 & 1 & 0 & 1 & 1 & 0 & 0 & 0 & 0 & 0 \\
\hline $\begin{array}{l}\text { Prawica } \\
\text { Rzeczypospolitej }\end{array}$ & 0 & 0 & 0 & 1 & 0 & 0 & 0 & 0 & 1 & 0 & 0 & 0 & 0 \\
\hline andere & 3 & 3 & 3 & 1 & 3 & 2 & 2 & 1 & 2 & 2 & 2 & 2 & 3 \\
\hline schwer zu sagen & 23 & 27 & 26 & 26 & 21 & 26 & 26 & 25 & 24 & 27 & 27 & 24 & 20 \\
\hline
\end{tabular}

Anm.: Berücksichtigt wurden die Antworten derer, die angaben, zur Wabl gehen zu wollen.

*Unterstützung für PJN

PO - Platforma Obywatelska/Bürgerplattform; PiS - Prawo i Sprawiedliwoś́l/Recht und Gerechtigkeit; PSL - Polskie Stronnictwo Ludowe/Polnische Bauernpartei; SLD -Sojusz Lewicy Demokratycznej/Demokratische Linksallianz; Nowa Prawica Janusza KorwinMikke - Neue Rechte von Janusz Korwin-Mikke; Solidarna Polska Zbigniewa Ziobra - Solidarisches Polen von Zbigniew Ziobro; Ruch Palikota/Palikot-Bewegung; PJN - Polska Jest Najważniejsza/Polen ist am Wichtigsten/Polska Razem Jarostawa Gowina; PPP - Polska Partia Pracy/Polnische Arbeitspartei; Prawica Rzeczypospolitej- Rechte der Republik

Quelle: CBOS Nr. 85/2014 Preferencje partyjne po wyborach do parlamentu europejskiego [Parteipräferenzen nach der Wahl zum Europäischen Parlament]. Warszawa 06/2014. <www.cbos.pl> 
Tabelle 6: Vertrauen zu Politikern im Zeitverlauf (\%)

\begin{tabular}{|c|c|c|c|c|c|c|c|c|c|c|c|c|c|}
\hline & $06 / 13$ & 07/13 & $08 / 13$ & $09 / 13$ & $10 / 13$ & $11 / 13$ & $12 / 13$ & $01 / 14$ & $02 / 14$ & $03 / 14$ & $04 / 14$ & $05 / 14$ & $06 / 14$ \\
\hline $\begin{array}{l}\text { Jarosław Gowin } \\
\text { (Vorsitzender von } \\
\text { Polska Razem } \\
\text { Jarosława Gowina) }\end{array}$ & 32 & 29 & 30 & 32 & 30 & - & - & 28 & 26 & 32 & 26 & 27 & 24 \\
\hline $\begin{array}{l}\text { Jarosław Kaczyński } \\
\text { (Vorsitzender von } \\
\text { PiS) }\end{array}$ & 34 & 32 & 30 & 33 & 31 & 31 & 30 & 35 & 31 & 31 & 29 & 32 & 34 \\
\hline $\begin{array}{l}\text { Bronisław } \\
\text { Komorowski } \\
\text { (Staatspräsident) }\end{array}$ & 72 & 71 & 71 & 73 & 69 & 71 & 69 & 71 & 71 & 75 & 70 & 70 & 77 \\
\hline $\begin{array}{l}\text { Janusz Korwin- } \\
\text { Mikke (Vor- } \\
\text { sitzender von Nowa } \\
\text { Prawica Janusza } \\
\text { Korwin-Mikke) }\end{array}$ & - & - & - & - & - & - & - & - & - & - & - & - & 19 \\
\hline $\begin{array}{l}\text { Leszek Miller } \\
\text { (Parteivorsitzender } \\
\text { der SLD) }\end{array}$ & 32 & 35 & 34 & 34 & 34 & 38 & 35 & 36 & 33 & 35 & 36 & 35 & 32 \\
\hline $\begin{array}{l}\text { Janusz Palikot } \\
\text { (Parteivorsitzender } \\
\text { von Ruch Palikota) }\end{array}$ & 20 & 18 & 18 & 27 & 22 & 24 & 19 & 21 & 23 & 21 & 19 & 19 & 17 \\
\hline $\begin{array}{l}\text { Janusz Piechociński } \\
\text { (Wirtschafts- } \\
\text { minister, Parteivor- } \\
\text { sitzender der PSL) }\end{array}$ & 24 & 25 & 25 & 25 & 24 & 23 & 25 & 27 & 27 & 28 & 24 & 30 & 27 \\
\hline $\begin{array}{l}\text { Donald Tusk } \\
\text { (Ministerpräsident, } \\
\text { Parteivorsitzender } \\
\text { der PO) }\end{array}$ & 34 & 32 & 31 & 34 & 29 & 32 & 31 & 34 & 35 & 41 & 34 & 36 & 40 \\
\hline
\end{tabular}

Polska Razem Jarostawa Gowina/Polen Gemeinsam von Jarosław Gowin

PiS - Prawo i SprawiedliwośćlRecht und Gerechtigkeit

Nowa Prawica Janusza Korwin-Mikke/Neue Rechte von Janusz Korwin-Mikke

SLD - Sojusz Lewicy Demokratycznej/Demokratische Linksallianz

Ruch Palikota/Palikot-Bewegung

PSL - Polskie Stronnictwo Ludowe/Polnische Bauernpartei

PO - Platforma Obywatelska/Bürgerplattform

Quelle: CBOS BS/89/2014 Zaufanie do polityków na początku czerwca [Vertrauen gegenüber Politikern Anfang Juni]. Warszawa 06/2014. <www.cbos.pl> 
Tabelle 7: Misstrauen gegenüber Politikern im Zeitverlauf (\%)

\begin{tabular}{|c|c|c|c|c|c|c|c|c|c|c|c|c|c|}
\hline & $06 / 13$ & $07 / 13$ & $08 / 13$ & $09 / 13$ & $10 / 13$ & $11 / 13$ & $12 / 13$ & $01 / 14$ & $02 / 14$ & $03 / 14$ & $04 / 14$ & $05 / 14$ & $06 / 14$ \\
\hline $\begin{array}{l}\text { Jarosław Gowin } \\
\text { (Vorsitzender von } \\
\text { Polska Razem } \\
\text { Jarosława Gowina) }\end{array}$ & 22 & 20 & 27 & 24 & 22 & - & - & 26 & 28 & 27 & 26 & 28 & 25 \\
\hline $\begin{array}{l}\text { Jarosław Kaczyński } \\
\text { (Vorsitzender von } \\
\text { PiS) }\end{array}$ & 46 & 45 & 50 & 47 & 48 & 48 & 50 & 49 & 48 & 48 & 50 & 48 & 46 \\
\hline $\begin{array}{l}\text { Bronisław } \\
\text { Komorowski } \\
\text { (Staatspräsident) }\end{array}$ & 11 & 12 & 12 & 12 & 13 & 11 & 14 & 13 & 12 & 11 & 11 & 12 & 8 \\
\hline $\begin{array}{l}\text { Janusz Korwin- } \\
\text { Mikke (Vor- } \\
\text { sitzender von Nowa } \\
\text { Prawica Janusza } \\
\text { Korwin-Mikke) }\end{array}$ & - & - & - & - & - & - & - & - & - & - & - & - & 50 \\
\hline $\begin{array}{l}\text { Leszek Miller } \\
\text { (Parteivorsitzender } \\
\text { der SLD) }\end{array}$ & 33 & 31 & 34 & 31 & 31 & 28 & 30 & 32 & 31 & 34 & 30 & 32 & 30 \\
\hline $\begin{array}{l}\text { Janusz Palikot } \\
\text { (Parteivorsitzender } \\
\text { von Ruch Palikota) }\end{array}$ & 56 & 58 & 56 & 51 & 54 & 51 & 53 & 56 & 50 & 56 & 55 & 53 & 57 \\
\hline $\begin{array}{l}\text { Janusz Piechociński } \\
\text { (Wirtschafts- } \\
\text { minister, Parteivor- } \\
\text { sitzender der PSL) }\end{array}$ & 13 & 11 & 15 & 13 & 14 & 14 & 16 & 11 & 13 & 14 & 16 & 15 & 12 \\
\hline $\begin{array}{l}\text { Donald Tusk } \\
\text { (Ministerpräsident, } \\
\text { Parteivorsitzender } \\
\text { der PO) }\end{array}$ & 47 & 48 & 49 & 49 & 51 & 50 & 50 & 47 & 45 & 42 & 46 & 43 & 40 \\
\hline
\end{tabular}

Polska Razem Jarostawa Gowina/Polen Gemeinsam von Jarostaw Gowin

PiS - Prawo i Sprawiedliwość/Recht und Gerechtigkeit

Nowa Prawica Janusza Korwin-Mikke/Neue Rechte von Janusz Korwin-Mikke

SLD - Sojusz Lewicy Demokratycznej/Demokratische Linksallianz

Ruch Palikota/Palikot-Bewegung

PSL - Polskie Stronnictwo Ludowe/Polnische Bauernpartei

PO - Platforma Obywatelska/Bürgerplattform

Quelle: CBOS BS/89/2014 Zaufanie do polityków na poczatku czerwca [Vertrauen gegenüber Politikern Anfang Juni]. Warszawa 06/2014. <www.cbos.pl> 


\section{7. - 30. Juni 2014}

\begin{tabular}{|c|c|}
\hline 17.06 .2014 & $\begin{array}{l}\text { Der Generalsekretär der Demokratischen Linksallianz (Sojusz Lewicy Demokratycznej - SLD), Krzysztof } \\
\text { Gawkowski, teilt mit, dass die SLD beim Sejmmarschall einen Antrag auf eine Regierungsinformation über } \\
\text { die »Abhöraffäre« eingereicht hat. Die SLD fordere Ministerpräsident Donald Tusk auf, den Sejm um ein Ver- } \\
\text { trauensvotum für die Regierung zu ersuchen. Hintergrund sind illegal mitgeschnittene Gespräche hochrangi- } \\
\text { ger Politiker und Personen öffentlicher Ämter, die die Wochenzeitung »Wprost« veröffentlicht hat. }\end{array}$ \\
\hline 18.06 .2014 & $\begin{array}{l}\text { Die Ukraine hebt das Embargo für polnisches Schweinefleisch auf, das im Februar nach Auftreten von Fällen } \\
\text { der sogenannten Afrikanischen Schweinepest in Polen verhängt worden war. Ausgenommen ist die Woiwod- } \\
\text { schaft Podlachien (woj. podlaskie). }\end{array}$ \\
\hline 19.06 .2014 & $\begin{array}{l}\text { Generalstaatsanwalt Andrzej Seremet verteidigt auf einer Pressekonferenz die Beschlagnahmung von Material in } \\
\text { der Redaktion des Wochenmagazins »Wprost« durch die Agentur für Innere Sicherheit (Agencja Bezpieczeństwa } \\
\text { Wewnętrznego - ABW) am Vortag. Dabei soll es zu Handgreiflichkeiten zwischen Mitarbeitern der ABW und } \\
\text { von »Wprost« gekommen sein. Der Einsatz der ABW fand im Zusammenhang mit der Veröffentlichung von ille- } \\
\text { gal mitgeschnittenen Gesprächen von ranghohen Politikern und Personen öffentlicher Ämter in »Wprost« statt. }\end{array}$ \\
\hline 20.06 .2014 & $\begin{array}{l}\text { Das Finanzministerium gibt bekannt, dass die Staatsverschuldung Ende April 477, } 9 \text { Mrd. Zloty betrug und im } \\
\text { Vergleich zum Vormonat um 0,4\% gestiegen ist. Im Vergleich zu Dezember } 2013 \text { hat sie sich um 18,2\% verringert. }\end{array}$ \\
\hline 22.06 .2014 & $\begin{array}{l}\text { Nach Informationen des Vorsitzenden der Polnischen Eisenbahngesellschaft PKP Polskie Linie Kolejowe, } \\
\text { Remigiusz Paszkiewicz, wird die Gesellschaft in den Jahren } 2014 \text { bis } 2020 \text { zirka } 50 \text { Mrd. Zloty in die Moder- } \\
\text { nisierung und den Bau neuer Trassen und Bahnhöfe investieren. Die Zufinanzierung aus EU-Mitteln beträgt } \\
\text { zirka } 40 \text { Mrd. Zloty. Modernisiert werden sollen u. a. Strecken zwischen Niederschlesien bzw. Oberschlesien } \\
\text { und Stettin, Gdingen und Danzig, die eine Schlüsselbedeutung für den Güterverkehr haben. }\end{array}$ \\
\hline 23.06 .2014 & $\begin{array}{l}\text { Ministerpräsident Donald Tusk teilt mit, dass er personelle Konsequenzen im Zuge der »Abhöraffäre« ablehnt. } \\
\text { Ziel der Abhöraktion sei es gewesen, den Staat in einer kritischen Phase Europas und der Ukraine zu lähmen. } \\
\text { Hintergrund sind illegale Mitschnitte von Gesprächen von Politikern und anderen Personen aus öffentlichen } \\
\text { Ämtern, die in der Wochenzeitung "Wprost« veröffentlicht worden waren. U. a. wurden Äußerungen von Außen- } \\
\text { minister Radosław Sikorski veröffentlicht, in denen er sich abfällig zu dem Zustand des polnisch-US-amerika- } \\
\text { nischen Bündnisses äußerte und dem britischen Premierminister David Cameron Europakompetenz absprach. }\end{array}$ \\
\hline 24.06 .2014 & $\begin{array}{l}\text { Die führenden Politiker der Regierungskoalition aus Bürgerplattform (Platforma Obywatelska-PO) und Pol- } \\
\text { nischer Bauernpartei (Polskie Stronnictwo Ludowe-PSL) thematisieren die sogenannte Abhöraffäre. Eine Pres- } \\
\text { sekonferenz findet anschließend nicht statt. Am Vortag hat Wirtschaftsminister und Vize-Ministerpräsident } \\
\text { Janusz Piechociński (PSL) von Ministerpräsident Donald Tusk gefordert, einen Termin zu setzen, bis zu dem } \\
\text { aufgeklärt sein müsse, wer die Aufnahmen der Gespräche von Politikern angefertigt hat, die in der Wochenzei- } \\
\text { tung »Wprost« veröffentlicht worden waren. Sollten die Umstände der illegalen Aufnahmen bis zum Ende der } \\
\text { Sommerpause nicht aufgeklärt sein, sollte eine neue Regierung eingesetzt werden. }\end{array}$ \\
\hline 25.06 .2014 & $\begin{array}{l}\text { Der Sejm spricht der Regierung von Ministerpräsident Donald Tusk mit } 237 \text { Stimmen gegen } 203 \text { Stimmen sein } \\
\text { Vertrauen aus. Der Fraktionsvorsitzende von Recht und Gerechtigkeit (Prawo i Sprawiedliwość - PiS), Mariusz } \\
\text { Błaszczak, kündigt einen Antrag auf ein konstruktives Misstrauensvotum an. Hintergrund ist die »Abhöraf- } \\
\text { färe«, d. h. die Veröffentlichung illegal mitgeschnittener Gespräche polnischer Politiker und Inhaber öffentli- } \\
\text { cher Ämter im Wochenmagazin „Wprost«. }\end{array}$ \\
\hline 26.06 .2014 & $\begin{array}{l}\text { In einem Gespräch mit der Tageszeitung "Rzeczpospolita» über die sogenannte Abhöraffäre fordert Jarosław } \\
\text { Gowin, Vorsitzender von Polen Gemeinsam von Jarosław Gowin (Polska Razem Jarosława Gowina), ehema- } \\
\text { liges Mitglied der Bürgerplattform (Platforma Obywatelska - PO) und ehemaliger PO-Justizminister, eine } \\
\text { Regierungsumbildung und anschließend vorgezogene Neuwahlen. Den Medienvertretern sei kein Vorwurf zu } \\
\text { machen, dass die Gesprächsmitschnitte von Politikern in der Wochenzeitung "Wprost« veröffentlicht wurden. } \\
\text { Es müssten aber die eventuellen Auftraggeber gefunden werden, um ein minimales Vertrauen der Polen in den } \\
\text { Staat wiederherzustellen. }\end{array}$ \\
\hline 27.06 .2014 & $\begin{array}{l}\text { Der Pressesprecher der Europäischen Kommission teilt mit, dass José Manuel Barroso, Präsident der Europäischen } \\
\text { Kommission, die Kandidatur des Vize-Staatssekretärs im polnischen Finanzministerium, Jacek Dominik, für das } \\
\text { Amt des EU-Haushaltskommissars angenommen hat. Dominik soll Nachfolger von Janusz Lewandowski werden. }\end{array}$ \\
\hline 29.06 .2014 & $\begin{array}{l}\text { Im Rahmen der Kampagne "Zeit für Veränderung«von Recht und Gerechtigkeit (Prawo i Sprawiedliwość - } \\
\text { PiS) kündigt Parteichef Jarosław Kaczyński Bemühungen an, die Rechte zu vereinigen. Die Regierung müsse } \\
\text { infolge der »Abhöraffäre« ihr Amt aufgeben, so Kaczyński. Die Kampagne organisiert offene Treffen aus Anlass } \\
\text { der Veröffentlichung von illegal mitgeschnittenen Gesprächen von Politikern. }\end{array}$ \\
\hline
\end{tabular}


\begin{tabular}{|l|l|}
\hline 30.06.2014 & $\begin{array}{l}\text { Nach Informationen der Polnischen Presseagentur (Polska Agencja Prasowa - PAP) hat der Minister für Indus- } \\
\text { trie und Handel der Tschechischen Republik, Jan Mladek, einen Brief an EU-Energiekommissar Günther } \\
\text { Oettinger und EU-Wettbewerbskommissar Joaquín Almunio gerichtet, in dem er dazu auffordert, die Atom- } \\
\text { energie nicht zu diskriminieren, sondern ihre wichtige Bedeutung für Europa anzuerkennen. Der Brief wurde } \\
\text { im Namen anderer west- und ostmitteleuropäischer Länder, u. a. Polen, verfasst. }\end{array}$ \\
\hline
\end{tabular}

Sie können die gesamte Chronik seit 2007 auch auf <http://www.laender-analysen.de/polen/> unter dem Link »Chronik«lesen. 
Die Polen-Analysen erscheinen zweimal monatlich als E-Mail-Dienst. Sie werden gemeinsam vom Deutschen PolenInstitut Darmstadt, der Bremer Forschungsstelle Osteuropa und der Deutschen Gesellschaft für Osteuropakunde in Partnerschaft mit dem Willy Brandt Zentrum für Deutschland- und Europastudien an der Universität Wroclaw (Breslau) herausgegeben.

Ein Archiv der Polen-Analysen finden Sie im Internet unter <www.laender-analysen.de/polen>

Kostenloses Abonnement unter <http://www.deutsches-polen-institut.de/Newsletter/subscribe.php>

Diese Analysen finden Sie online als Lizenzausgabe auf $<$ bpb.de>

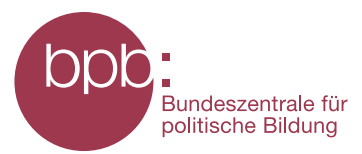

\section{Deutsches Polen-Institut Darmstadt}

Das Deutsche Polen-Institut Darmstadt (DPI) ist ein Forschungs-, Informations-, und Veranstaltungszentrum für polnische Kultur, Geschichte, Politik, Gesellschaft und die deutsch-polnischen Beziehungen, die sich im Kontext der europäischen Integration entwickeln. Das seit März 1980 aktive und bis 1997 von Gründungsdirektor Karl Dedecius geleitete Institut ist eine Gemeinschaftsgründung der Stadt Darmstadt, der Länder Hessen und Rheinland-Pfalz sowie des Bundes. 1987 wurden die Kultusminister der Länder und 2011 das Auswärtige Amt weitere institutionelle Träger. Einen wesentlichen Beitrag zur Verwirklichung der Institutsziele leisten private Stiftungen. Das DPI hat satzungsgemäß die Aufgabe, durch seine Arbeit zur Vertiefung der gegenseitigen Kenntnisse des kulturellen, geistigen und gesellschaftlichen Lebens von Polen und Deutschen beizutragen.

Ziel der Vermittlertätigkeit des DPI ist es, „die zu interessieren, auf die es politisch, wirtschaftlich, gesellschaftlich und kulturell im deutsch-polnischen Verhältnis ankommt« (Leitlinien 1997). Es geht um die Entscheider und Multiplikatoren in Politik, Kultur, Bildung, Verwaltung, Medien und Wirtschaft und, wesentlich stärker ausgeprägt als bisher, um das Hineinwirken in Wissenschaft, Forschung und Bildung.

Derzeit bemüht sich das DPI in Kooperation mit den verstreuten Orten wissenschaftlicher Polen-Kompetenz an deutschen Hochschulen und Forschungsinstituten verstärkt darum, ausgehend von einer Bestandsaufnahme deutscher Polen-Forschung Ort wissenschaftlicher Forschung und verbindendes, vernetzendes und kooperierendes Zentrum zu werden. Ausgangspunkt der Neuausrichtung ist die kaum mehr kontrollierbare Dynamik des Rückbaus der Ressourcen der wissenschaftlichen Polen-Kompetenz in den unterschiedlichen Disziplinen. Mit der über 60.000 Bände zählenden multidisziplinären Fachbibliothek für Polen, die eine einzigartige Sammlung polnischer Literatur in der Originalsprache und in deutscher Übersetzung umfasst, ist das DPI bereits ein geschätzter Ort der Recherche und des wissenschaftlichen Arbeitens. (www.deutsches-polen-institut.de)

Forschungsstelle Osteuropa an der Universität Bremen (www.forschungsstelle.uni-bremen.de)

1982 gegründet, widmet sich die Forschungsstelle Osteuropa an der Universität Bremen der interdisziplinären Analyse der Länder Ost- und Ostmitteleuropas in Zeitgeschichte und Gegenwart. Der Forschungsschwerpunkt liegt dabei auf der Rolle von »Dissens und Konsens«, von Opposition und Zivilgesellschaft in ihrem historischen, politischen, gesellschaftlichen und kulturellen Kontext. Die Forschungsstelle besitzt in ihrem Archiv eine einzigartige Sammlung alternativer Kulturgüter und unabhängiger Texte aus den ehemaligen sozialistischen Ländern. Darunter befindet sich auch eine umfangreiche Sammlung des "Zweiten Umlaufs«, die das Schrifttum und Dokumente unabhängiger Initiativen und gesellschaftlicher Gruppen in Polen aus der Zeit von 1976 bis zum Umbruch umfasst. Hinzu kommt eine umfangreiche Bibliothek mit wissenschaftlicher Literatur. Mit Archiv, Bibliothek und zwei wissenschaftlichen Abteilungen ist die Forschungsstelle auch eine Anlaufstelle sowohl für Gastwissenschaftler als auch für die interessierte Öffentlichkeit.

Eine der Hauptaufgaben der Forschungsstelle ist die Information der interessierten Öffentlichkeit. Dazu gehören unter anderem regelmäßige E-Mail-Informationsdienste für Politik, Wirtschaft, Zivilgesellschaft und Medien.

\section{Herausgegeben mit finanzieller Unterstützung der Stiftung für deutsch-polnische Zusammenarbeit}

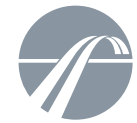

FUNDACJA WSPÓEPRACY

STIFTUNG

STIFTUNG
FUR DEUTSCH-POLNISCHE
ZUSAMMENARBEIT

Die Meinungen, die in den Polen-Analysen geäußert werden, geben ausschließlich die Auffassung der Autoren wieder. Abdruck und sonstige publizistische Nutzung sind nach Rücksprache mit der Redaktion gestattet. Redaktion: Prof. Dr. Dieter Bingen (verantwortlich) (Darmstadt), Silke Plate, M.A. (Bremen) Technische Gestaltung: Matthias Neumann

Polen-Analysen-Layout: Cengiz Kibaroglu, Matthias Neumann

Alle Ausgaben der Polen-Analysen sind mit Themen- und Autorenindex archiviert unter www.laender-analysen.de

Die Polen-Analysen werden im Rahmen der Datenbank World Affairs Online (WAO) ausgewertet und sind im Portal IREON www.ireon-portal.de recherchierbar.

ISSN 1863-9712 @ 2014 by Deutsches Polen-Institut Darmstadt und Forschungsstelle Osteuropa, Bremen

Kontakt: Dr. Andrzej Kaluza, Presse- und Öffentlichkeitsarbeit, Deutsches Polen-Institut, Mathildenhöhweg 2.

D-64287 Darmstadt, Tel.: 06151/4985-13, Fax: 06151/4985-10, E-Mail: polen-analysen@dpi-da.de, Internet: www.laender-analysen.de/polen 